\title{
Evolution of cytokinesis-related protein localization during the emergence of multicellularity in volvocine green algae
}

Yoko Arakaki ${ }^{1}$, Takayuki Fujiwara², Hiroko Kawai-Toyooka ${ }^{1}$, Kaoru Kawafune ${ }^{1,3}$, Jonathan Featherston ${ }^{4,5}$, Pierre M. Durand ${ }^{4,6}$, Shin-ya Miyagishima² and Hisayoshi Nozaki ${ }^{*}$ (D)

\begin{abstract}
Background: The volvocine lineage, containing unicellular Chlamydomonas reinhardtii and differentiated multicellular Volvox carteri, is a powerful model for comparative studies aiming at understanding emergence of multicellularity. Tetrabaena socialis is the simplest multicellular volvocine alga and belongs to the family Tetrabaenaceae that is sister to more complex multicellular volvocine families, Goniaceae and Volvocaceae. Thus, T. socialis is a key species to elucidate the initial steps in the evolution of multicellularity. In the asexual life cycle of C. reinhardtii and multicellular volvocine species, reproductive cells form daughter cells/colonies by multiple fission. In embryogenesis of the multicellular species, daughter protoplasts are connected to one another by cytoplasmic bridges formed by incomplete cytokinesis during multiple fission. These bridges are important for arranging the daughter protoplasts in appropriate positions such that species-specific integrated multicellular individuals are shaped. Detailed comparative studies of cytokinesis between unicellular and simple multicellular volvocine species will help to elucidate the emergence of multicellularity from the unicellular ancestor. However, the cytokinesis-related genes between closely related unicellular and multicellular species have not been subjected to a comparative analysis.

Results: Here we focused on dynamin-related protein 1 (DRP1), which is known for its role in cytokinesis in land plants. Immunofluorescence microscopy using an antibody against T. socialis DRP1 revealed that volvocine DRP1 was localized to division planes during cytokinesis in unicellular C. reinhardtii and two simple multicellular volvocine species T. socialis and Gonium pectorale. DRP1 signals were mainly observed in the newly formed division planes of unicellular C. reinhardtii during multiple fission, whereas in multicellular T. socialis and G. pectorale, DRP1 signals were observed in all division planes during embryogenesis.

Conclusions: These results indicate that the molecular mechanisms of cytokinesis may be different in unicellular and multicellular volvocine algae. The localization of DRP1 during multiple fission might have been modified in the common ancestor of multicellular volvocine algae. This modification may have been essential for the re-orientation of cells and shaping colonies during the emergence of multicellularity in this lineage.
\end{abstract}

Keywords: Multicellularity, Volvocine algae, Tetrabaena socialis, DRP1

\footnotetext{
*Correspondence: nozaki@bs.s.u-tokyo.ac.jp

1 Department of Biological Sciences, Graduate School of Science, University of

Tokyo, 7-3-1 Hongo, Bunkyo-ku, Tokyo 113-0033, Japan

Full list of author information is available at the end of the article
} 


\section{Background}

The transition to multicellularity is one of the most compelling events during the evolution of life and has occurred more than 25 times in distinct eukaryotic lineage [1]. To fully understand these transitions comparative biological studies are essential. Major complex multicellular groups such as metazoans and land plants emerged from unicellular ancestors that existed approximately 600 to 1000 million years ago [2]. There are few extant species that represent the initial features of multicellular ancestors or transitional forms from unicellular to multicellular within the lineages closely related to metazoans and land plants. In contrast, the multicellular volvocine green algae (Fig. 1) diverged from a unicellular ancestor only 200 million years ago [3]. In addition, there are many extant species representing various steps in the transition to multicellularity, which makes the volvocines unique as a model lineage.

The volvocine lineage (Fig. 1) includes unicellular Chlamydomonas reinhardtii, undifferentiated multicellular species like Tetrabaena socialis and Gonium pectorale, and differentiated multicellular species such as Volvox carteri [3-5]. The simplest multicellular species is four-celled T. socialis belonging to the Tetrabaenaceae, which is sister to the large clade composed of the remaining, more complex colonilal/multicellular volvocine algae (Goniaceae and Volvocaceae) (Fig. 1) [5-7]. T. socialis shares at least four common features with more complex multicellular volvocine members: incomplete cytokinesis, rotation of basal bodies, transformation of the cell wall to extracellular matrix, and modulation of cell number $[3,5]$. Recently, whole nuclear genome analyses of G. pectorale [8] suggested that modifications of cell cycle regulation genes (duplication of cyclin D1 gene and alterations in the retinoblastoma protein) occurred in the common ancestor of
G. pectorale and $V$. carteri and that these modifications were the basis for genetic modulation of cell number. However, there is less information about more downstream molecules that actually participate in the formation of integrated multicellular individuals during embryogenesis.

During the asexual life cycles of unicellular and multicellular volvocine algae, reproductive cells perform successive divisions (rapid S/M phase alternating without G2 phase) known as multiple fission $[9,10]$. The unicellular species $C$. reinhardtii forms $2^{\mathrm{n}}$ (n: number of rounds of cell divisions) daughter cells depending on the size of the mother cell [11], whereas reproductive cells of multicellular volvocine members form $2^{\mathrm{n}}$ daughter protoplasts that are regulated by mother cell size and genetic control [4]. These daughter protoplasts are connected to one another by cytoplasmic bridges, which are important for the arrangement of cells within the daughter colony of multicellular volvocine algae [4] like T. socialis [5], G. pectorale [12] and V. carteri [13]. Considering that both of these multicellular member-specific traits (modulation of daughter cell number and incomplete cytokinesis) are recognized in the tetrabaenacean species $T$. socialis, comparative molecular analyses of multiple fission between unicellular and multicellular forms is essential to understand the initial steps to multicellularity in this lineage.

Various cytokinesis-related genes are characterized in metazoans [14] and land plants [15]. Modes of cytokinesis are variable: mother cells divide into two daughter cells by fission in metazoans, whereas two daughter cells are produced by a cell plate newly formed in land plant mother cells [16]. However, there are some common molecules associated with cytokinesis in metazoans and land plants $[14,15]$ such as dynamin-related protein

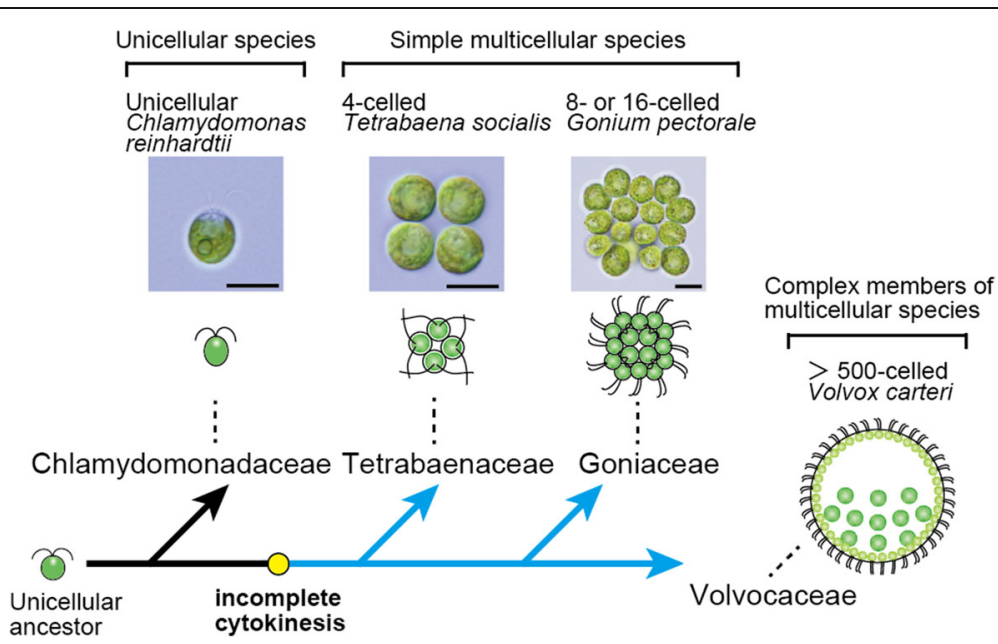

Fig. 1 Simplified representation of volvocine phylogeny and evolution of incomplete cytokinesis, focusing the three volvocine species examined in the present study. The phylogeny is based on previous studies [3, 6, 7]. Scale bars: $10 \mu \mathrm{m}$ 
(DRP). Dynamin was originally described as a microtubulebinding protein that was isolated from bovine brain extracts [17]. Dynamin homologs are often categorized as classical or conventional dynamins, which have five distinct domains: GTPase domain, middle domain, pleckstrin-homology domain, GTPase effecter domain (GED), and proline-rich domain [18, 19]. The dynamin superfamily contains additional members that lack a pleckstrin-homology domain and/or proline-rich domain, or have additional domains that are not present in classical dynamins. These members are defined as dynaminrelated proteins (DRPs) [19]. In metazoans and land plants, several dynamins and DRPs play important roles in cytokinesis [18, 19]. For example, in Arabidopsis thaliana, DRP1A and DRP1E are localized in the cell plate [20], DRP2B is co-localized with DRP1A in the cell plate during cytokinesis and functions in vesicle formation [21], and DRP5A is localized in the cell plate at the end of cell division [22]. The double mutant line drp1a/drple of $A$. thaliana is unable to accomplish the embryogenesis because of defects in cell wall formation [20].

In this study, we focused on the DRP1 homologs in the volvocine lineage to examine the contribution of cytokinesis-related genes to the initial stages of multicellularity. We determined the complete coding region of the DRP1 homolog of the simplest multicellular species $T$. socialis (TsDRP1), and performed immunofluorescence microscopy with a newly raised anti-TsDRP1 antibody in unicellular $C$. reinhardtii and two colonial multicellular species T. socialis and G. pectorale (Fig. 1).

\section{Methods}

\section{Strains and culture conditions}

Three algal strains were used in this study: $C$. reinhardtii strain cw92 (CC-503, cell-wall deficient, distributed by the Chlamydomonas Resource Center) [23], T. socialis strain NIES-571 [5], and G. pectorale strain 2014-0520-F1-4 (a sibling strain of plus and minus strains used previously [24]). C. reinhardtii strain cw92 was cultured synchronously in $300 \mathrm{~mL}$ tris-acetate-phosphate medium [25] in a silicon-capped $500 \mathrm{~mL}$ flask with aeration at $25{ }^{\circ} \mathrm{C}$, on a light: dark cycle $12 \mathrm{~h}: 12 \mathrm{~h}$ under cool-white fluorescent lamps at an intensity of $110-150 \mu \mathrm{mol} \cdot \mathrm{m}^{-2} \cdot \mathrm{s}^{-1}$. T. socialis strain NIES-571 was cultured synchronously in $300 \mathrm{~mL}$ standard Volvox medium [26] in a silicon-capped $500 \mathrm{~mL}$ flask with aeration at $20{ }^{\circ} \mathrm{C}$, on a light: dark cycle $12 \mathrm{~h}$ : $12 \mathrm{~h}$ under cool-white fluorescent lamps at an intensity of $110-150 \mu \mathrm{mol} \cdot \mathrm{m}^{-2} \cdot \mathrm{s}^{-1}$ [5]. G. pectorale strain 2014-0520F-4 was cultured synchronously in $300 \mathrm{~mL}$ standard Volvox medium [26] in a silicon-capped $500 \mathrm{~mL}$ flask with aeration at $20{ }^{\circ} \mathrm{C}$, on a light: dark cycle $12 \mathrm{~h}: 12 \mathrm{~h}$ under cool-white fluorescent lamps at an intensity of 130$180 \mu \mathrm{mol} \cdot \mathrm{m}^{-2} \cdot \mathrm{s}^{-1}$. To evaluate the synchrony, percentages of dividing cells were monitored 15-16 times during $24 \mathrm{~h}$.

\section{Identification of TsDRP1 gene}

To determine the complete coding region of TsDRP1, partial coding sequences were obtained from our ongoing $T$. socialis strain NIES-571 genome assembly by a TBLASTN search using $C$. reinhardtii DRP1 (CrDRP1) as a query, and TsDRP1 specific primers (Additional file 1: Table S1) were designed based on the sequences. Polyadenylated mRNA of $T$. socialis was isolated with Dynabeads oligo $(\mathrm{dT})_{25}$ (Thermo Fisher Scientific, Waltham, MA, USA) and reverse transcribed with Superscript III reverse transcriptase (Thermo Fisher Scientific) [24]. PCR was carried out using the synthesized cDNA as follows: $94{ }^{\circ} \mathrm{C}$ for $1 \mathrm{~min}, 35$ cycles of $94{ }^{\circ} \mathrm{C}$ for $30 \mathrm{~s}, 60{ }^{\circ} \mathrm{C}$ for $30 \mathrm{~s}$, and $72{ }^{\circ} \mathrm{C}$ for $2 \mathrm{~min}$, followed by $72{ }^{\circ} \mathrm{C}$ for 5 min with TaKaRa LA-Taq with GC Buffer (Takara Bio Inc., Otsu, Japan) by using a thermal cycler GeneAmp PCR System 9700 (Thermo Fisher Scientific). Amplified DNA was purified with illustra GFX PCR DNA and Gel Band Purification Kits (GE Healthcare, Buckinghamshire, UK) and sequenced directly by an ABI PRISM 3100 Genetic Analyzer (Thermo Fisher Scientific) using a BigDye Terminator cycle sequencing ready reaction kit, v.3.1 (Thermo Fisher Scientific). The 5' and 3' ends of TsDRP1 were determined by RACE using the GeneRacer ${ }^{\mathrm{TM}}$ kit (Thermo Fisher Scientific) according to the manufacturer's protocol. Each synthesized RACE product was amplified with KOD FX Neo DNA polymerase (TOYOBO, Osaka, Japan). PCR was carried out as follows: $94{ }^{\circ} \mathrm{C}$ for $2 \mathrm{~min}, 5$ cycles of $98{ }^{\circ} \mathrm{C}$ for $10 \mathrm{~s}$ and $74{ }^{\circ} \mathrm{C}$ for $1.5 \mathrm{~min}, 5$ cycles of $98{ }^{\circ} \mathrm{C}$ for $10 \mathrm{~s}$ and $72{ }^{\circ} \mathrm{C}$ for $1.5 \mathrm{~min}, 5$ cycles of $98{ }^{\circ} \mathrm{C}$ for $10 \mathrm{~s}$ and $70{ }^{\circ} \mathrm{C}$ for $1.5 \mathrm{~min}, 15$ cycles of $98{ }^{\circ} \mathrm{C}$ for $10 \mathrm{~s}$ and $68{ }^{\circ} \mathrm{C}$ for $1.5 \mathrm{~min}$, and followed by $68{ }^{\circ} \mathrm{C}$ for $7 \mathrm{~min}$. Amplified DNA was purified and sequenced as described above. Domains of DRP1proteins were searched by using Pfam program (http://pfam.xfam.org) [27].

\section{Phylogenetic analyses}

The amino acid sequences of DRP1 and DRP2 from six streptophytes (land plants and Klebsormidium flaccidum) and six chlorophytes (Additional file 1: Table S2) were collected from the genome databases in Phytozome (https:// phytozome.jgi.doe.gov/pz/portal.html) and National Center for Biotechnology Information (https://www.ncbi.nlm. nih.gov/) by BLASTP and TBLASTN searches [28] using CrDRP1 and A. thaliana DRP2A as queries and aligned with the newly determined TsDRP1 by MAFFTv7 [29] server (http://mafft.cbrc.jp/alignment/server/). Phylogenetic analyses were performed using the maximum-likelihood and neighbor joining methods with PhyML [30] and MEGA 5.2.2 programs [31], respectively. The LG + I + G + F model was selected using ProtTest3 [32] optimized using Akaike information criteria. The bootstrap analyses were performed with 1000 replicates. Because DRP1 clade 
is sister to DRP2 clade [22], DRP2 sequences were treated as the outgroup in the present study.

\section{Preparation of antibodies}

The antibody against $T$. socialis DRP1 (anti-TsDRP1 antibody) was raised in rabbits using the recombinant polypeptide. The cDNA sequence encoding the protein (corresponding to 11-597 positions of TsDRP1 amino acids; Additional file 2: Figure S1) was amplified by PCR using the primers listed in Additional file 1: Table S1. The PCR product was cloned into a pET100 expression vector (Thermo Fisher Scientific) and $6 x$ His fusion polypeptide was expressed in Rosetta (DE3) Escherichia coli cells, purified using a HisTrap HP column (GE healthcare). The purified polypeptide was subjected to SDS-PAGE, and gel slices containing the recombinant polypeptide were homogenized and injected into rabbits for antibody production (Kiwa Laboratory Animals. Co., Ltd., Wakayama, Japan). Antibodies were affinitypurified from the antisera by using the recombinant polypeptide coupled to a HiTrap NHS-activated HP column (GE Healthcare). Evaluation of the specificity of the anti-TsDRP1 antibody in $T$. socialis, G. pectorale and $C$. reinhardtii was performed by western blot analyses as described below (Additional file 2: Figure S2, Information S1).

\section{Western blot analyses}

Expression of DRP1 proteins in the three volvocine species was analyzed by SDS-PAGE and western blot modified from a previous study [33]. Cells were harvested, suspended in SDS-sample buffer (100 mM dithiothreitol, $2 \%$ SDS, $10 \%$ glycerol, $0.005 \%$ Bromophenol blue in $62.5 \mathrm{mM}$ Tris- $\mathrm{HCl}$ ) and boiled for three minutes. The prepared samples were separated on an Any kD MiniPROTEAN TGX precast gel (Bio-Rad, Hercules, CA, USA) and transferred onto a Hybond-P membrane (GE Healthcare, Uppsala, Sweden). The blotted membrane was blocked with $3 \%$ skim milk in TPBS [0.1\% Tween 20 (Sigma Aldrich) in phosphate-buffered saline (PBS)] at $4{ }^{\circ} \mathrm{C}$ overnight. The blot was incubated with an antiTsDRP1 antibody diluted 1: 2000 with 3\% skim milk in TPBS for $1 \mathrm{~h}$ at room temperature and washed in 3\% skim milk in TPBS. The membrane was incubated with a goat anti-rabbit IgG antibody conjugated to horseradish peroxidase (Jackson ImmunoResearch, WestGrove, PA, USA) diluted 1:2000 with 3\% skim milk in TPBS for $1 \mathrm{~h}$ at room temperature and washed with TPBS. The protein signals were detected with Amersham ECL prime Western blotting detection reagent (GE Healthcare). Images were obtained by ChemiDoc XRS system (Bio-Rad) with Quantity One software (Bio-Rad).

To examine the expression of DRP1 at the protein level, five time-course samples were obtained from each synchronous culture of C. reinhardtii, T. socialis, and G. pectorale: a sample with the greatest number of dividing cells during $24 \mathrm{~h}$ ( 0 point), three hours before the 0 point ( -3 point), six hours before the 0 point ( -6 point), three hours after the 0 point ( +3 point), and six hours after the 0 point ( 6 point). The time-course samples were analyzed by SDS-PAGE and western blot as described above. For Coomassie brilliant blue staining as loading control, each duplicated SDS-PAGE gel was stained by EzStain AQua (ATTO, Tokyo, Japan).

\section{Indirect immunofluorescence microscopy}

Fixation of $C$. reinhardtii was performed using a modified method in a previous study [34]. C. reinhardtii cells were attached to polyethyleneimine coated coverslips and fixed in $-20{ }^{\circ} \mathrm{C}$ methanol for $5 \mathrm{~min}$, transferred to fresh $-20{ }^{\circ} \mathrm{C}$ methanol for $5 \mathrm{~min}$ and air-dried. The dried cells were incubated in PBS for $10 \mathrm{~min}$. Subsequent blocking and antibody reactions were performed based on a previous study [5]. Immunostaining of $T$. socialis and G. pectorale were performed as described previously [5]. The anti-TsDRP1 antibody and a monoclonal anti-tubulin alpha antibody (clone YL1/2, BioRad, Hercules, CA, USA), used as primary antibodies, were diluted 1: 500 with blocking buffer $(0.11 \%$ Gelatin [Sigma Aldrich], $0.05 \% \mathrm{NaN}_{3}, 0.25 \%$ bovine serum albumin [Sigma Aldrich] in TPBS). Alexa Fluor 488 goat anti-rabbit IgG (H + L) (\# A11008, Invitrogen, Carlsbad, CA, USA) and Alexa Fluor 568 goat anti-rat $\operatorname{IgG}(\mathrm{H}+\mathrm{L})$ (\# A11077, Invitrogen) were also diluted 1: 500 with the blocking buffer. Confocal and differential interference contrast (DIC) images were obtained with an FV-1200 (Olympus, Tokyo, Japan) and three serial images were merged by using Adobe Photoshop CS6 software (Adobe Systems Inc., San Jose, CA, US).

\section{Results \\ Identification and characterization of TsDRP1}

The full-length coding region of TsDRP1 (1890 bp) was determined and the deduced amino acid sequence (629 amino acids) aligned with DRP1 homologs of $A$. thaliana (AtDRP1A), C. reinhardtii (CrDRP1), G. pectorale (GpDRP1), and V. carteri (VcDRP1) (Additional file 2: Figure S1). The DRP1 sequences were highly conserved within the volvocine algae: the identity of TsDRP1 with CrDRP1, GpDRP1, and VcDRP1 was 92\%, 92\%, and 91\%, respectively. The GC contents of the volvocine $D R P 1$ coding region were higher (CrDRP1: 64.18\%, TSDRP1: 65.12\%, GPDRP1: $62.22 \%$, and VCDRP1: 57.55\%) than that of AtDRP1A (46.78\%), which is consistent with their GC-rich genome compositions $[8,23,35]$. Three domains that characterize DRP1, GTPase domain, middle domain, and GED [19], were found in all DRP1 sequences of volvocine algae as well as AtDRP1A. The motifs for interactions to 
GTP (G1-4) [18] were conserved in AtDRP1A and volvocine DRP1 sequences (Additional file 2: Figure S1).

To confirm that DRP1 sequences of volvocine algae are orthologous to the DRP1 proteins of land plants, phylogenetic analyses were performed. All five land plants possessed several paralogs of DRP1 genes, whereas each of the green algae (six chlorophytes and streptophyte $K$. flaccidum) possessed a single DRP1 gene in the nuclear genome. The DRP1 clade was subdivided into two monophyletic groups corresponding to streptophytes and chlorophytes (Fig. 2). However, while the streptophyte clade was robustly resolved (with 92-100\% bootstrap values), the monophyly of the chlorophytes was much less supported (with 60\% bootstrap values in only maximum-likelihood method). The chlorophytes were composed of two robust clades, Chlorophyceae/ Trebouxiophyceae and Mamiellophyceae with 99-100\% bootstrap values. Within the former clade, four chlorophycean or volvocine DRP1 sequences formed a robust monophyletic group with $100 \%$ bootstrap values. These results were consistent with the phylogeny using multiple chloroplast genes [36-38], and indicate that DRP1 genes of volvocine algae are orthologs of DRP1 of streptophytes.

\section{Expression patterns of CrDRP1, TsDRP1 and GpDRP1 during the asexual life cycle}

To examine the relationship between volvocine DRP1 and cytokinesis at a protein expression level, we performed western blot analyses using the anti-TsDRP1 antibody.

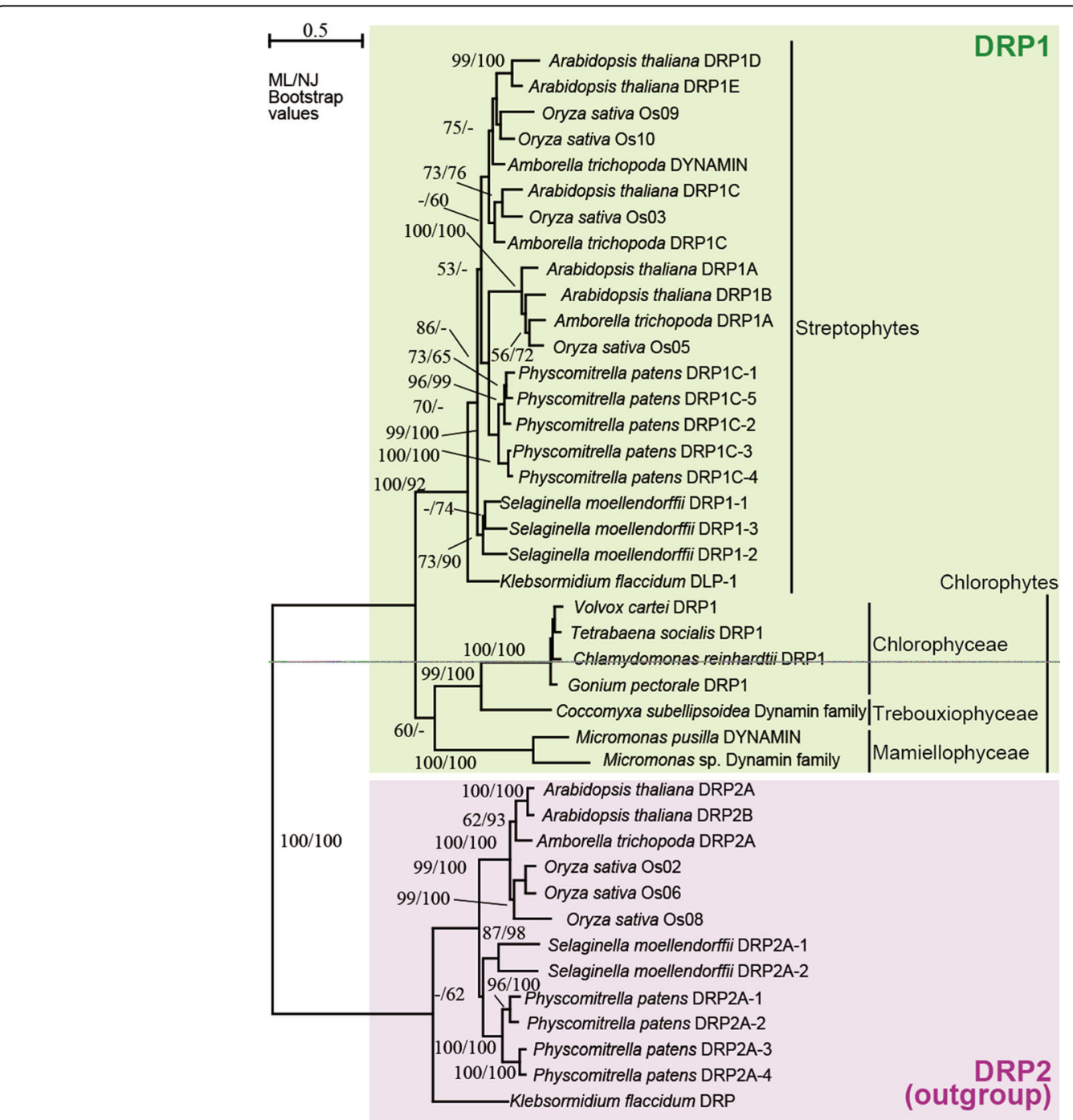

Fig. 2 Maximum-likelihood (ML) tree of dynamin-related proteins. Bootstrap values ( $\geq 50 \%)$ for the $M L$ and neighbor-joining (NJ) analyses are indicated left and right side, respectively. DRP2 sequences were used as outgroup. The scale bar corresponds to 0.5 amino acid substitutions per position 
The CrDRP1, TsDRP1, and GpDRP1 signals were detected as major bands at $\sim 75 \mathrm{kDa}$ with the anti-TsDRP1 antibody (Additional file 2: Figure S2). DRP1 signals were constitutively detected from all time-course samples of the three species (Additional file 2: Figure S3).

\section{Subcellular localization of DRP1 during the asexual life cycle}

To verify the subcellular localization of DRP1, immunofluorescence microscopy was carried out using the antiTsDRP1 and anti-tubulin alpha antibodies. Immunofluorescences of tubulin were used as the division plane marker, because microtubule structures (phycoplast) are observed in division planes of volvocine algae [39-41]. In vegetative cells of $C$. reinhardtii, T. socialis and $G$. pectorale, DRP1 signals were observed as many speckles in their cytoplasm (Figs. 3a-d, 4a-d, 5a-d). In two-celled stage, microtubules were observed in the cleavage furrows and DRP1 signals were localized in the vicinity of the division plane of $C$. reinhardtii (white arrowheads in Fig. 3e-h), T. socialis (white arrowheads in Fig. 4e-h) and G. pectorale (white arrowheads in Fig. 5e-h). While tubulin and DRP1 signals were not perfectly co-localized in the two-celled stage, these appeared to be partially overlapped (Figs. 3h, 4h, 5h). The DRP1 fluorescence in cytoplasm at this stage was less than that of vegetative cell (Figs. 3f, 4f, 5f).
DRP1 localizations of the four-celled stage were different between the unicellular and multicellular species examined here. In the four-celled stage of C. reinhardtii, DRP1 was mainly localized in second division planes (Fig. 3i-l). In contrast, DRP1 in four-celled embryos of $T$. socialis and G. pectorale were observed clearly in both first and second division planes (Figs. 4i-l, 5i-l). Eightcelled embryo of G. pectorale also exhibited clear DRP1 localization in the first, second and third division planes (Fig. 5m-p).

\section{Discussion}

In C. reinhardtii, T. socialis and G. pectorale, DRP1 signals were mainly observed in their cytoplasm during the vegetative phase (Figs. 3b, 4b, 5b). However, the signals were mainly accumulated in the division plane during multiple fission of the three volvocine species (Figs. 3f, j, $4 f, j, 5 f, j, n)$. DRP1 expression was always detected and did not drastically change during the time courses examined in the three species (Additional file 2: Figure S3). Thus, volvocine DRP1 might change localization during the life cycle. The volvocine DRP1 localizations in the division plane were similar to those of some DRP1 proteins of land plants: DRP1A (ADL1) in A. thaliana [20] and tobacco BY-2 cells [42], and DRP1C in tobacco BY-2 cells [42]. Therefore, DRP1 proteins of volvocine algae may act as cytokinesis-related molecules.

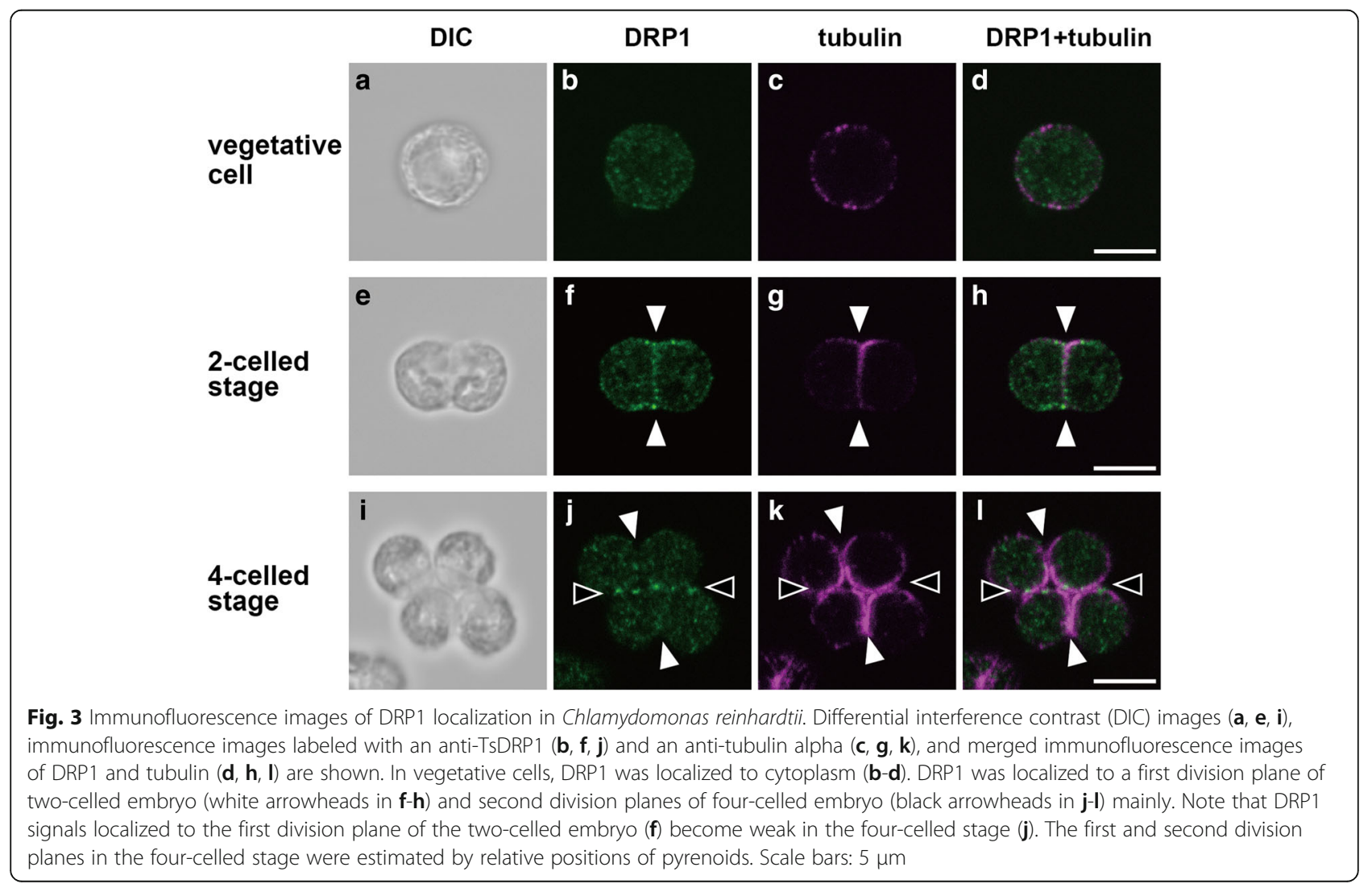




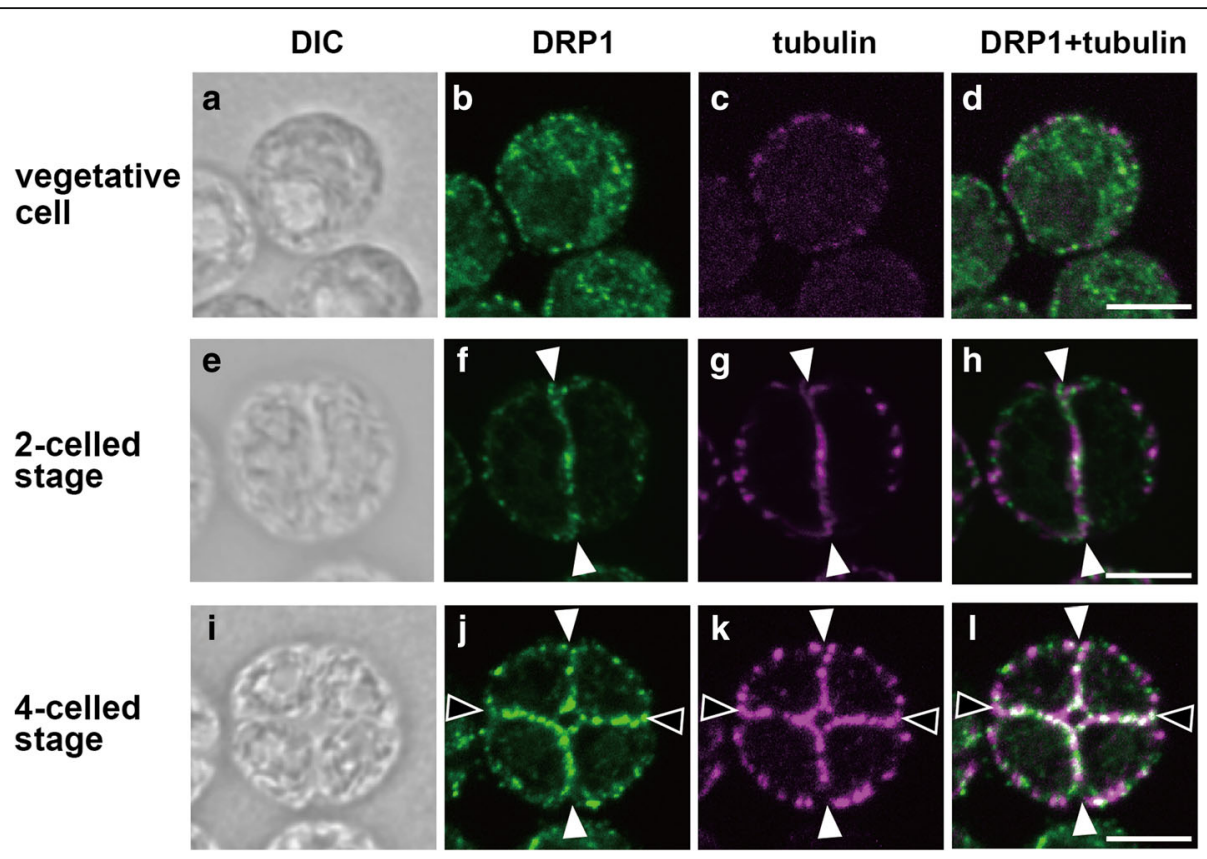

Fig. 4 Immunofluorescence images of DRP1 localization in Tetrabaena socialis. Differential interference contrast (DIC) images (a, e, i), immunofluorescence images labeled with an anti-TsDRP1 $(\mathbf{b}, \mathbf{f}, \mathbf{j})$ and an anti-tubulin alpha $(\mathbf{c}, \mathbf{g}, \mathbf{k})$, and merged immunofluorescence images of DRP1 and tubulin ( $\mathbf{d}, \mathbf{h}, \mathbf{l})$ are shown. In vegetative cells, DRP1 was localized to cytoplasm (b-d). DRP1 was localized to a first division plane (white arrowheads in $\mathbf{f}-\mathbf{h}$ ) of two-celled embryo and both first (white arrowheads in $\mathbf{j}-\mathbf{l}$ ) and second (blank arrowheads in $\mathbf{j}-\mathbf{l}$ ) division planes of four-celled embryo. The first and second division planes in the four-celled stage were estimated by directions of the planes within the parental colony. Scale bars: $10 \mu \mathrm{m}$

In the second division of multiple fission (4-celled stage), DRP1 of $C$. reinhardtii was mainly localized in the second division plane (Fig. 3j), while DRP1 signals of T. socialis (Fig. 4j) and G. pectorale (Fig. 5j) were clearly observed in the both division planes, which were formed by the first and second divisions. Moreover, in the third division of G. pectorale (8-celled stage), DRP1 signals were observed in all division planes (Fig. 5n). Thus, DRP1 may have a role mainly in last division plane of unicellular C. reinhardtii (Fig. 3f, j), whereas DRP1 may equally work in all division planes of multicellular $T$. socialis and G. pectorale (Figs. 4f, j,5f, j, n). Each cell division during multiple fission of multicellular $T$. socialis [5], G. pectorale [12], and other multicellular volvocine algae [13, 43-45] proceeds through incomplete cytokinesis and the resulting daughter protoplasts are connected to one another by cytoplasmic bridges, whereas daughter protoplasts of $C$. reinhardtii are completely separated from one another by means of complete cytokinesis $[9,10]$. The division planes of multicellular volvocine species are therefore different from those of unicellular C. reinhardtii at subcellular and molecular levels.

Dynamins and DRPs are involved in various membrane remodeling events such as budding and trafficking of vesicles, fission or fusion of organelles, and cytokinesis [22, 46]. Particularly in cytokinesis, dynamins are associated with newly formed membranes of metazoans such as Caenorhabditis elegans [47] and zebrafish [48]. In Dictyostelium discoideum, the relationships between cytokinesis and dynamin A [49], dynamin-like protein (Dlp) A, DlpB and DlpC [22] were reported. In A. thaliana, DRP1A [20], DRP1E [20], DRP2B [21], and DRP5A [22] are localized in the division plane, and function in cytokinesis. Those dynamins and DRPs play important roles for cytokinesis such as membrane fission and vesicle formation for cytokinesis. Therefore, volvocine DRP1 proteins might be related to membrane remodeling during the multiple fission. Hence, the few DRP1 signals in the first division plane of $C$. reinhardtii 4-celled stage (Fig. 3j) may indicate that membrane fission/formation mediated by DRP1 has been finished in this plane after the first division (white arrowheads in Fig. $3 \mathrm{j}$ ), but has been occurring at the second division plane (black arrowheads in Fig. 3j). The presence of DRP1 signals in all division planes of T. socialis (Fig. 4j) and G. pectorale (Fig. 5j, n) may be due to the continuous membrane remodeling during the incomplete cytokinesis in these two multicellular species.

\section{Conclusion}

This study demonstrated that localization patterns of DRP1 are different between unicellular C. reinhardtii and two multicellular species T. socialis and G. pectorale. 


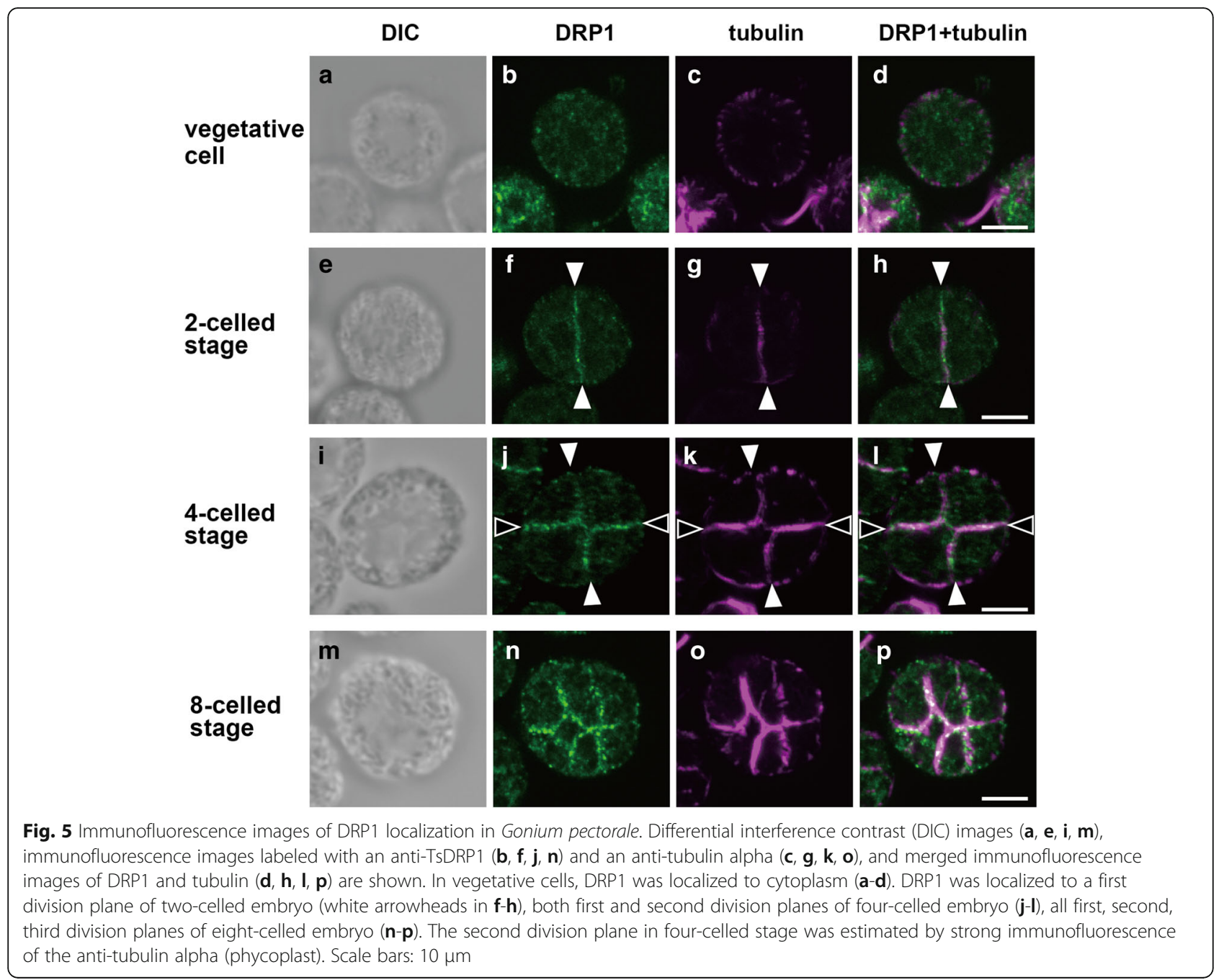

Given that DRP1 may function in volvocine cytokinesis, the different DRP1 localization patterns between $C$. reinhardtii and two multicellular species $T$. socialis and G. pectorale (Fig. 6) indicate differences in the molecular mechanisms of cytokinesis during multiple fission between unicellular forms and multicellular forms. $T$. socialis and G. pectorale are considered to represent ancestral (plesiomorphic) multicellular morphology based on the cladistic analysis of morphological data and molecular phylogenetic analyses (Fig. 1) [3-7]. These data indicate therefore, that the localization patterns of DRP1 during multiple fission might have been modified from unicellular Chlamydomonas-type to multicellular Tetrabaena/Gonium-type (Fig. 6) in the

\section{Unicellular \\ Chlamydomonas-type}

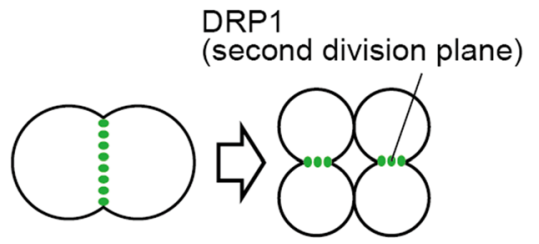

\section{Multicellular \\ Tetrabaena/Gonium-type}

DRP1

(all division planes)
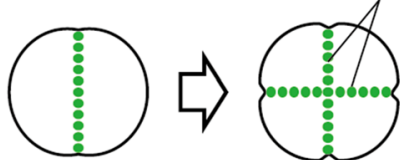

Fig. 6 Schematic diagram of DRP1 localization patterns in volvocine algae. In unicellular Chlamydomonas reinhardtii, DRP1 is mainly localized to second division planes of four-celled stage (unicellular Chlamydomonas-type). In multicellular Tetrabaena socialis and Gonium pectorale, DRP1 is localized to all division planes of four-celled embryo (multicellular Tetrabaena/Gonium-type) 
common ancestor of multicellular volvocine algae (Fig. 1). This modification was essential for the initial stages of colonial living in this lineage.

\section{Additional files}

Additional file 1 Table S1. List of primers used for amplification and sequencing of TSDRP1. Table S2. List of DRP1 and DRP2 proteins used in this study. (PDF $84 \mathrm{~kb}$ )

Additional file 2 Figure S1. Alignment of DRP1A from

Arabidopsis thaliana (At) and DRP1 from Chlamydomonas reinhardtii (Cr), Tetrabaena socialis (Ts), Gonium pectorale (Gp) and Volvox carteri (Vc). Black and gray background indicates identical or similar amino acid, respectively. GTPase domain, dynamin middle domain, and GTPase effector domain are indicated by pink, green, and yellow background color, respectively. The region corresponding to the antigen for an antiTsDRP1 antibody is showed under the alignment (gray bar). Figure S2. Specificity of the affinity-purified anti-TsDRP1 antibody. The specificity of the anti-TsDRP1 antibody was validated in three volvocine algae by western blotting. A single band was detected in each lane ( $75 \mathrm{kDa})$ with the antibody that was incubated with acetone powder of $E$. coli with the empty vector (left) while no signal was detected with the antibody that was incubated with acetone powder of E. coli expressing TsDRP1 (middle). For details of the methods, see Information S1 (Additional file 2). Figure S3. Western blot analyses of DRP1 proteins of Chlamydomonas reinhardtii (CrDRP1), Tetrabaena socialis (TsDRP1), and Gonium pectorale (GpDRP1) using anti-TsDRP1 antibody. Time-course of synchronous culture and western blot (WB) of C. reinhardtii, T. socialis, and G. pectorale are shown in $\mathbf{a}, \mathbf{b}$, and $\mathbf{c}$, respectively. Time-course samples were obtained from five points (arrows in each line graph): the greatest number of dividing cells $(0)$, three $(-3)$ and six $(-6)$ hours before 0 point, and three $(+3)$ and six $(+6)$ hours after 0 point. Coomassie brilliant blue (CBB) staining of a duplicate gel shows the equal protein loading in each lane. Information S1. Methods for specificity of the affinity-purified anti-TsDRP1 antibody (Additional file 2: Figure S2). (PDF $1785 \mathrm{~kb}$ )

\section{Abbreviations}

DIC: Differential interference contrast; Dlp: Dynamin-like protein; DRP: Dynamin-related protein; GED: GTPase effector domain; GTP: Guanosine triphosphate; GTPase: Guanosine triphosphatease; PBS: Phosphate-buffered saline; TPBS: 0.1\% Tween 20 in PBS

\section{Acknowledgements}

We would like to thank Dr. Kenji Kimura (National Institute of Genetics) for fluorescence observation using the confocal laser microscopy.

\section{Funding}

This work was supported by NIG-JOINT (2016-B to HN), Grants-in-Aid for JSPS Fellows (No. 25-9234 to YA) and Scientific Research (A) (grant number $16 \mathrm{H} 02518$ to HN) from MEXT/JSPS KAKENHI, and the National Research Foundation, South Africa (grant number RA151217156515 to PD).

\section{Availability of data and materials}

The newly determined TsDRP1 sequence has been deposited to DDBJ/EMBL/ GenBank (accession number: LC279615). The sequence alignment used for phylogenetic analyses of DRP1 (Fig. 2) has been deposited in TreeBASE (https:// treebase.org/treebase-web/home.html; study ID: S21468). Gonium pectorale strain 2014-0520-F1-4 is available from Microbial Culture Collection at the National Institute for Environmental Studies (http://mcc.nies.go.jp/ localeAction.do?lang=en) as NIES-4121. All the other data generated or analyzed during this study are included in this published article and its Additional files 1 and 2 .

\section{Authors' contributions}

YA, HKT, KK, SM and HN conceived and designed the experiments. YA, JF, PD, and HN constructed the draft genome assembly of Tetrabaena. YA, TF and SM performed the experiments and analyzed the data. YA and HN wrote the manuscript. All authors read and approved the manuscript.
Ethics approval and consent to participate

Not applicable.

\section{Consent for publication}

Not applicable.

\section{Competing interests}

The authors declare that they have no competing interests.

\section{Publisher's Note}

Springer Nature remains neutral with regard to jurisdictional claims in published maps and institutional affiliations.

\section{Author details}

'Department of Biological Sciences, Graduate School of Science, University of Tokyo, 7-3-1 Hongo, Bunkyo-ku, Tokyo 113-0033, Japan. Department of Cell Genetics, National Institute of Genetics, 1111 Yata, Mishima, Shizuoka 411-8540, Japan. ${ }^{3}$ Department of Life Science and Technology, School of Life Science and Technology, Tokyo Institute of Technology, 2-12-1 Ookayama, Meguro-ku, Tokyo 152-8550, Japan. ${ }^{4}$ Evolutionary Studies Institute, University of the Witwatersrand, Johannesburg 2000, South Africa. ${ }^{5}$ Agricultural Research Council, Biotechnology Platform, Pretoria 0040, South Africa. ${ }^{6}$ Department of Ecology and Evolutionary Biology, University of Arizona, Tucson, AZ 85721, USA.

Received: 16 August 2017 Accepted: 24 November 2017 Published online: 06 December 2017

\section{References}

1. Grosberg RK, Strathmann RR. The evolution of multicellularity: a minor major transition? Annu Rev Ecol Evol Syst. 2007;38:621-54.

2. Sharpe SC, Eme L, Brown MW, Roger AJ. Timing the origins of multicellular eukaryotes through Phylogenomics and relaxed molecular clock analyses. In: Trillo IR, Nedelcu AM, editors. Evolutionary transitions to multicellular life. Dordrecht: Springer Netherlands; 2015. p. 3-29.

3. Herron MD, Hackett JD, Aylward FO, Michod RE. Triassic origin and early radiation of multicellular volvocine algae. Proc Natl Acad Sci U S A. 2009; 106:3254-8.

4. Kirk DLA. Twelve-step program for evolving multicellularity and a division of labor. BioEssays. 2005;27:299-310.

5. Arakaki Y, Kawai-Toyooka H, Hamamura Y, Higashiyama T, Noga A, Hirono $\mathrm{M}$, et al. The simplest integrated multicellular organism unveiled. PLoS One. 2013;8:e81641

6. Nozaki H, Ito M. Phylogenetic relationships within the colonial Volvocales (Chlorophyta) inferred from cladistic analysis based on morphological data. J Phycol. 1994;30:353-65.

7. Nozaki H, Misawa K, Kajita T, Kato M, Nohara S, Watanabe MM. Origin and evolution of the colonial Volvocales (Chlorophyceae) as inferred from multiple, chloroplast gene sequences. Mol Phylogenet Evol. 2000;17:256-68.

8. Hanschen ER, Marriage TN, Ferris PJ, Hamaji T, Toyoda A, Fujiyama A, et al. The Gonium pectorale genome demonstrates co-option of cell cycle regulation during the evolution of multicellularity. Nat Commun. 2016;7: 11370 .

9. Harris EH. The Chlamydomonas sourcebook: introduction to Chlamydomonas and its laboratory use. 2nd ed. Oxford: Academic Press; 2009.

10. Kirk DL. Volvox: molecular-genetic origins of multicellularity and cellular differentiation. Cambridge: Cambridge University Press; 1998.

11. Umen JG, Goodenough UW. Control of cell division by a retinoblastoma protein homolog in Chlamydomonas. Genes Dev. 2001;15:1652-61.

12. lida H, Ota S, Inouye I. Cleavage, incomplete inversion, and cytoplasmic bridges in Gonium pectorale (Volvocales, Chlorophyta). J Plant Res. 2013; 126:699-707

13. Green KJ, Viamontes Gl, Kirk DL. Mechanism of formation, ultrastructure, and function of the cytoplasmic bridge system during morphogenesis in Volvox. J Cell Biol. 1981:91:756-69.

14. Eggert US, Mitchison TJ, Field CM. Animal cytokinesis: from parts list to mechanisms. Annu Rev Biochem. 2006;75:543-66.

15. Jürgens G. Plant cytokinesis: fission by fusion. Trends Cell Biol. 2005;15:277-83.

16. Field C, Li R, Oegeme K. Cytokinesis in eukaryotes: a mechanistic comparison. Curr Opin Cell Biol. 1999;11:68-80. 
17. Shpetner HS, Vallee RB. Identification of dynamin, a novel mechanochemical enzyme that mediates interactions between microtubules. Cell. 1989;59: 421-32.

18. Praefcke GJK, McMahon HT. The dynamin superfamily: universal membrane tubulation and fission molecules? Nat Rev Mol Cell Biol. 2004;5:133-47.

19. Konopka CA, Schleede JB, Skop AR, Bednarek SY. Dynamin and cytokinesis. Traffic. 2006;7:239-47.

20. Kang B-H, Busse JS, Bednarek SY. Members of the Arabidopsis dynamin-like gene family, ADL1, are essential for plant cytokinesis and polarized cell growth. Plant Cell. 2003;15:899-913.

21. Fujimoto M, Arimura S, Ueda T, Takahashi H, Hayashi Y, Nakano A, et al. Arabidopsis dynamin-related proteins DRP2B and DRP1A participate together in clathrin-coated vesicle formation during endocytosis. Proc Natl Acad Sci U S A. 2010;107:6094-9.

22. Miyagishima S, Kuwayama H, Urushihara H, Nakanishi H. Evolutionary linkage between eukaryotic cytokinesis and chloroplast division by dynamin proteins. Proc Natl Acad Sci U S A. 2008;105:15202-7.

23. Merchant SS, Prochnik SE, Vallon O, Harris EH, Karpowicz SJ, Witman GB, et al. The Chlamydomonas genome reveals the evolution of key animal and plant functions. Science. 2007;318:245-51.

24. Kawai-Toyooka H, Mori T, Hamaji T, Suzuki M, Olson BJSC, Uemura T, et al. Sex-specific posttranslational regulation of the gamete fusogen GCS1 in the isogamous volvocine alga Gonium pectorale. Eukaryot Cell. 2014;13:648-56.

25. Gorman DS, Levine RP. Cytochrome $f$ and plastocyanin: their sequence in the photosynthetic electron transport chain of Chlamydomonas reinhardtii. Proc Natl Acad Sci U S A. 1965;54:1665-9.

26. Kirk DL, Kirk MM. Protein synthetic patterns during the asexual life cycle of Volvox carteri. Dev Biol. 1983;96:493-506.

27. Finn RD, Coggill P, Eberhardt RY, Eddy SR, Mistry J, Mitchell AL, et al. The Pfam protein families database: towards a more sustainable future. Nucleic Acids Res. 2016;44:D279-85.

28. Altschul SF, Madden TL, Schäffer AA, Zhang J, Zhang Z, Miller W, Lipman DJ, Gapped BLAST. PSI-BLAST: a new generation of protein database search programs. Nucleic Acids Res. 1997;25:3389-402.

29. Katoh K, Standley DMMAFFT. Multiple sequence alignment software version 7: improvements in perforemance and usability. Mol Biol Evol. 2013;30:772-80

30. Guindon S, Dufayard JF, Lefort V, Anisimova M, Hordijk W, Gascuel O. New algorithms and methods to estimate maximum-likelihood phylogenies: assessing the performance of PhyML 3.0. Syst Biol. 2010;59:307-21.

31. Tamura K, Peterson D, Peterson N, Stecher G, Nei M, Kumar S. MEGA5: Molecular evolutionary genetics analysis using maximum likelihood, evolutionary distance, and maximum parsimony methods. Mol Biol Evol. 2011:28:2731-9.

32. Darriba D, Taboada GL, Doallo R, Posada D. ProtTest 3: fast selection of bestfit models of protein evolution. Bioinformatics. 2011;27:1164-5.

33. Nakazawa Y, Hiraki M, Kamiya R, Hirono M. SAS-6 is a cartwheel protein that establishes the 9-fold symmetry of the centriole. Curr Biol. 2007;17:2169-74.

34. Lechtreck K-F, Luro S, Awata J, Witman GB. HA-tagging of putative flagellar proteins in Chlamydomonas reinhardtii identifies a novel protein of intraflagellar transport complex B. Cell Motil Cytoskeleton. 2009;66:469-82.

35. Prochnik SE, Umen J, Nedelcu AM, Hallmann A, Miller SM, Nishii I, et al. Genome analysis of organismal complexity in the multicellular green alga Volvox carteri. Science. 2010;329:223-6.

36. Smith SA, Beaulieu JM, Donoghue MJ. Mega-phylogeny approach for comparative biology: an alternative to supertree and supermatrix approaches. BMC Evol Biol. 2009;9:37.

37. Leliaert F, Smith DR, Moreau H, Herron MD, Verbruggen $H$, Delwiche CF, Clerck OD. Phylogeny and molecular evolution of green algae. CRC Crit Rev Plant Sci. 2012;31:1-46.

38. Ruhfel BR, Gitzendanner MA, Soltis PS, Soltis D, Burleigh JG. From algae to angiosperms-inferring the phylogeny of green plants (Viridiplantae) from 360 plastid genomes. BMC Evol Biol. 2014;14:23.

39. Doonan $\mathrm{JH}$, Grief C. Microtubule cycle in Chlamydomonas reinhardtii: an immunofluorescence study. Cell Motil Cytoskeleton. 1987;7:381-92.

40. Kirk DL, Kaufman MR, Keeling RM, Stamer KA. Genetic and cytological control of the asymmetric divisions that pattern the Volvox embryo. Dev Suppl. 1991;1:67-82.

41. Dymek EE, Goduti D, Kramer T, Smith EFA. Kinesin-like calmodulin-binding protein in Chlamydomonas: evidence for a role in cell division and flagellar functions. J Cell Sci. 2006;119:3107-16.
42. Hong Z, Geisler-Lee CJ, Zhang Z, Verma DPS. Phragmoplastin dynamics: multiple forms, microtubule association and their roles in cell plate formation in plants. Plant Mol Biol. 2003;53:297-312.

43. Fulton AB. Colonial development in Pandorina morum. II. Colony morphogenesis and formation of the extracellular matrix. Dev Biol. 1978;64:236-51.

44. Marchant HJ. Colony formation and inversion in green alga Eudorina elegans. Protoplasma. 1977;93:325-39.

45. Iida H, Nishii I, Inouye I. Embryogenesis and cell positioning in Platydorina caudata (Volvocaceae, Chlorophyta). Phycologia. 2011;50:530-40.

46. Antonny B, Burd C, De Camilli P, Chen E, Daumke O, Faelber K, et al. Membrane fission by dynamin: what we know and what we need to know. EMBO J. 2016:35:2270-84.

47. Thompson HM, Skop AR, Euteneuer U, Meyer BJ, McNiven MA. The large GTPase dynamin a associates with the spindle midzoe and is required for cytokinesis. Curr Biol. 2002;12:2111-7.

48. Feng B, Schwarz H, Jesuthasan S. Furrow-specific endocytosis during cytokinesis of zebrafish blastomeres. Exp Cell Res. 2002;279:14-20.

49. Wienke DC, Knetsch MLW, Neuhaus EM, Reedy MC, Manstein DJ. Disruption of a dynamin homologue affects endocytosis, organelle morphology, and cytokinesis in Dictyostelium discoideum. Mol Biol Cell. 1999;10:225-43.

\section{Submit your next manuscript to BioMed Central and we will help you at every step:}

- We accept pre-submission inquiries

- Our selector tool helps you to find the most relevant journal

- We provide round the clock customer support

- Convenient online submission

- Thorough peer review

- Inclusion in PubMed and all major indexing services

- Maximum visibility for your research

Submit your manuscript at www.biomedcentral.com/submit
) Biomed Central 